\title{
Permanence of a stochastic delay competition model with Lévy jumps
}

\author{
Meng Liu ${ }^{\mathrm{a}, \mathrm{b}, *}$, Meiling Deng ${ }^{\mathrm{a}}$, Zhaojuan Wang ${ }^{\mathrm{a}}$ \\ a School of Mathematical Science, Huaiyin Normal University, Huaian 223300, P. R. China. \\ ${ }^{b}$ School of Mathematics and Statistics, Northeast Normal University, Jilin 130024, P. R. China.
}

Communicated by D. Baleanu

\begin{abstract}
Permanence is one of the most important topics in biomathematics. The question of permanence of stochastic multi-species models is challenging because the current approaches can not be used. In this paper, an asymptotic approach is used, and sufficient criteria for permanence of a general n-species stochastic delay Lotka-Volterra competition model with Lévy jumps are established. It is also shown that these criteria are sharp in some cases. The results reveal that the stochastic noises play a key role in the permanence. This approach can be also applied to investigate the permanence of other stochastic population models with/without time delay and/or Lévy noises. (C)2017 All rights reserved.
\end{abstract}

Keywords: Permanence, stochastic perturbations, delay.

2010 MSC: 92D25, 60H10, 60H30.

\section{Introduction}

Permanence, indicating the long-term survival of all species in a model, has regarded as one of the most important concepts in ecology and epidemics. The concept of permanence was originally proposed in late 1970s for ordinary differential equations (ODEs), and then were developed rapidly in the 1980s to difference systems, functional differential equations (FDEs) and partial differential equations (PDEs) [36]. Now, it has become one of the most important topics in biomathematics [27].

In the natural world, the growth of species is inevitably affected by environmental perturbations $[7,8,21,22,24,25,28,46]$. The concept of permanence is also extended to stochastic models and attracts much attention [27,35]. There are two widely used definitions of permanence for stochastic population models. The first one is as follows:

Definition 1.1 ([17]). Let $N(t)=\left(N_{1}(t), \cdots, N_{n}(t)\right)^{\top}$ stand for the solution of a stochastic population model. If for arbitrary $\varepsilon \in(0,1)$, there are positive constants $\alpha_{1}=\alpha_{1}(\varepsilon)$ and $\alpha_{2}=\alpha_{2}(\varepsilon)$ such that

$$
\liminf _{t \rightarrow \infty}\left\{|N(t)|=\sqrt{\sum_{i=1}^{n} N_{i}^{2}(t)} \geqslant \alpha_{1}\right\} \geqslant 1-\varepsilon, \liminf _{t \rightarrow \infty}\left\{|N(t)| \leqslant \alpha_{2}\right\} \geqslant 1-\varepsilon,
$$

then the model is said to be stochastically permanent (which we refer to as SP1).

\footnotetext{
*Corresponding author

Email address: liumeng0557@sina.com (Meng Liu)
}

doi:10.22436/jnsa.010.06.36 
Using this definition,

- Li and Mao [17] considered the following non-autonomous stochastic competitive system

$$
d N_{i}(t)=N_{i}(t)\left(r_{i}(t)-\sum_{j=1}^{n} c_{i j}(t) N_{j}(t)\right) d t+\sigma_{i}(t) N_{i}(t) d W_{i}(t), \quad i=1, \cdots, n,
$$

where $N_{i}(t)$ represents the size of the $i$-th species at time $t, r_{i}(t)>0, c_{i j}(t) \geqslant 0, \sigma_{i}(t)$ are all continuous and bounded functions defined on $[0,+\infty),\left\{\left(W_{1}(t), \ldots, W_{n}(t)\right)\right\}_{t \geqslant 0}$ is an $n$-dimensional Brownian motion defined on a complete probability space $\left(\Omega,\left\{\mathcal{F}_{t}\right\}_{t \in R_{+}}, P\right)$ with a filtration $\left\{\mathcal{F}_{t}\right\}_{t \in R_{+}}$, $\sigma_{i}^{2}$ is the intensity of the stochastic noise. The authors [17] proved that if

$$
\min _{1 \leqslant i \leqslant n}\left\{\inf _{t \geqslant 0} c_{i i}(t)\right\}>0, \min _{1 \leqslant i \leqslant n}\left\{\inf _{t \geqslant 0} b_{i}(t)\right\}>0,
$$

where $b_{i}(t)=r_{i}(t)-0.5 \sigma_{i}^{2}(t)$, then model (1.1) is SP1.

- Li et al. [16, Theorem 3.3] analyzed the SP1 of stochastic Lotka-Volterra systems with Markovian switching.

- Bao et al. [3, Theorem 4.1] obtained the sufficient conditions for SP1 of a stochastic competitive model with Lévy jumps.

- Tran and Yin [38, Theorem 4.2] investigated the SP1 of stochastic competitive models with partial observation.

- Mandal et al. [31, Theorem 3.3.1] studied the SP1 of a allelopathic phytoplankton model.

- Tan et al. [37, Theorem 3.1] considered the SP1 of a stochastic competitive model with impulsive perturbations.

More results of SP1 for stochastic population models can be found in Li et al. [15, Theorem 3.3], Lv and Wang [29, Theorem 3.2], Qiu et al. [34, Theorem 6], Zhang and Wang [45, Theorem 6.2], Jiang et al. [12, Theorem 2.1], Lv and Wang [30, Theorem 3.5], Li et al. [14, Theorem 4.3].

However, Definition 1.1 is not appropriate in some cases. Definition 1.1 means

$$
\liminf _{t \rightarrow \infty} P\left\{\sqrt{\sum_{i=1}^{n} N_{i}^{2}(t)} \geqslant \alpha_{1}\right\} \geqslant 1-\varepsilon .
$$

This can not guarantee all the species have a positive lower bound, some species can go to extinction as long as one species has a positive lower bound. To see this more clearly, consider the following two-species competitive model:

$$
\left\{\begin{array}{l}
d N_{1}(t)=N_{1}(t)\left[0.6-0.3 N_{1}(t)-0.32 N_{2}(t)\right] d t+0.447 N_{1}(t) d W_{1}(t), \\
d N_{2}(t)=N_{2}(t)\left[0.55-0.28 N_{1}(t)-0.3 N_{2}(t)\right] d t+0.447 N_{2}(t) d W_{2}(t) .
\end{array}\right.
$$

According to [27], the species 2 in system (1.2) will go to extinction almost surely (a.s.), i.e., $\lim _{t \rightarrow \infty} N_{2}(t)=0$ a.s. However, by the work of Li and Mao [17], model (1.2) is SP1.

The other definition of permanence for stochastic population models was proposed by Schreiber et al. [35]. Consider the following stochastic differential equation (SDE)

$$
d N_{i}(t)=N_{i}(t)\left\{\rho_{i}(N(t)) d t+\sum_{j=1}^{m} \sigma_{i j}(N(t)) d W_{j}(t)\right\}, \quad i=1, \cdots, n, x(0) \in R_{+}^{n},
$$

where $N(t)=\left(N_{1}(t), \cdots, N_{n}(t)\right)^{\top}, R_{+}^{n}=\left\{\eta \in R^{n} \mid \eta_{i}>0,1 \leqslant i \leqslant n\right\}$. Let $\bar{R}_{+}^{n}=\left\{\eta \in R^{n} \mid \eta_{i} \geqslant 0,1 \leqslant i \leqslant n\right\}$ and $\Lambda_{0}=\left\{\eta \in \bar{R}_{+}^{n} \mid \eta_{i}=0\right.$ for some $\left.i, 1 \leqslant i \leqslant n\right\}$. 
Definition 1.2 ([35]). If there exists a unique invariant measure $\mu$ such that

(i) the distribution of $\mathrm{N}(\mathrm{t})$ converges to $\mu$ as $\mathrm{t} \rightarrow+\infty$, and

(ii) $\mu\left(\Lambda_{0}\right)=0$,

then model (1.3) is said to be stochastically persistent (which we refer to as SP2).

Clearly, SP2 can ensure the long-term survival of all species. At the same time, SP2 implies SP1, but not the converse.

Schreiber et al. [35, Theorem 4] have shown that if for all invariant measures $v$ supported on $\Lambda_{0}$, $\lambda_{*}(v):=\max _{1 \leqslant i \leqslant n} \lambda_{i}(v)>0$, where

$$
\lambda_{i}(v)=\int \lambda_{i}(N) v(d N), \quad \lambda_{i}(N)=\rho_{i}(N)-\frac{1}{2} q_{i i}(N), \quad q_{i j}(N)=\sum_{k=1}^{m} \sigma_{i k}(N) \sigma_{k j}(N),
$$

then the solution $N(t)$ of (1.3) is SP2. However, these results can not apply to investigate most stochastic population models. For example, consider the following stochastic logistic model:

$$
d N(t)=N(t)\left(r-\frac{N(t)}{K}\right) d t+\sigma N(t) d W(t), \quad N(0)>0 .
$$

By Remark 2.10 below, we can see that for model (1.4), $\lambda_{*}(v)=0$.

Then an interesting and important question arises: is there another approach to study the SP2 of stochastic population models? The main aim of this paper is to study this question. An asymptotic approach is used, and sufficient criteria for the SP2 of a stochastic delay competition model with Lévy jumps are established. We also show that these criteria are sharp in some cases. The results demonstrate that the stochastic perturbations play a key role in determining the SP2 of the models. At the end of this paper, we show that this approach can be also used to study the SP2 of other stochastic population models with/without time delay and/or Lévy noises, and as an example, we establish the sharp criteria for SP2 of a stochastic delay predator-prey model with Lévy jumps.

\section{Main results}

In this paper, we consider the following stochastic delay competitive model with Lévy jumps:

$$
\begin{aligned}
d N_{i}(t)= & N_{i}\left(t^{-}\right)\left\{\left(r_{i}-c_{i i} N_{i}\left(t^{-}\right)-\sum_{j=1, j \neq i}^{n} c_{i j} N_{j}\left(t^{-}-\tau_{i j}\right)\right) d t\right. \\
& \left.+\sigma_{i} d W_{i}(t)+\int_{\mathbb{Y}} \gamma_{i}(u) \tilde{\Gamma}(d t, d u)\right\}, \quad i=1, \cdots, n,
\end{aligned}
$$

with initial condition

$$
\mathrm{N}(\xi)=\left(\mathrm{N}_{1}(\xi), \cdots, \mathrm{N}_{\mathrm{n}}(\xi)\right)^{\mathrm{T}}=\left(\phi_{1}(\xi), \cdots, \phi_{\mathrm{n}}(\xi)\right)^{\mathrm{T}}=\phi(\xi) \in \Phi,
$$

where $N_{i}(t)$ is the size of species $i$ at time $t, N\left(t^{-}\right)$is the left limit of $N(t), r_{i}>0$ and $c_{i j}>0$ are constants representing the growth rate and competition coefficient respectively, $\Gamma(d t, d u)=\Gamma(d t, d u)-\theta(d u) d t, \Gamma$ is a Poisson counting measure, $\theta$ is the characteristic measure of $\Gamma, \mathbb{Y}$ is a subset of $(0,+\infty)$ with $\theta(\mathbb{Y})<+\infty$, $\tau_{i j} \geqslant 0$ represents the time delay, $i, j=1, \cdots, n, \tau=\max _{i, j=1, \ldots, n, j \neq i}\left\{\tau_{i j}\right\}, \Phi$ is the family of all continuous functions from $[-\tau, 0]$ to $R_{+}^{n}$. For biological reasons, we assume that [3]

$$
1+\gamma_{i}(u)>0, \quad u \in \mathbb{Y}, \quad i=1, \cdots, n .
$$


It is useful to point out that some special cases of (2.1) have been studied extensively in literature, for example, [11, 12, 15-17, 37, 38] considered model (2.1) with $\tau_{i j}=0$ and $\gamma_{i}(u)=0,[23,39,42]$ investigated model (2.1) with $\gamma_{i}(u)=0$. Model (2.1) with $\tau_{i j}=0$ was analyzed in [3].

Throughout this paper, as a standing hypothesis we assume that $\Gamma$ and $\left\{\left(W_{1}(t), \cdots, W_{n}(t)\right)\right\}_{t} \geqslant 0$ are independent. For simplicity, we introduce the following notations.

$$
\begin{aligned}
& b_{i}=r_{i}-0.5 \sigma_{i}^{2}-\int_{\mathbb{Y}}\left[\gamma_{i}(u)-\ln \left(1+\gamma_{i}(u)\right)\right] \theta(d u), \\
& f_{i}=b_{i}-\sum_{j=1, j \neq i}^{n} \frac{c_{i j}}{c_{j j}} b_{j}, \quad m_{i}(t)=\int_{0}^{t} \int_{\mathbb{Y}} \ln \left(1+\gamma_{i}(u)\right) \tilde{\Gamma}(d s, d u), \quad i=1, \cdots, n .
\end{aligned}
$$

Let $C=\operatorname{det}\left(\left(c_{i j}\right)_{n \times n}\right)$, let $C_{i}$ be the determinant obtained by changing the ith column of $C$ to $\left(b_{1}, b_{2}, \cdots, b_{n}\right)^{\top}$. By Golpalsamy [10], if $b_{i}>0$ and $f_{i}>0, i=1, \cdots, n$, then $\left(\frac{c_{1}}{C}, \cdots, \frac{c_{n}}{C}\right)^{\top}$ is a unique positive solution of the following equations

$$
\left\{\begin{array}{l}
c_{11} N_{1}+c_{12} N_{2}+\cdots+c_{1 n} N_{n}=b_{1} \\
c_{21} N_{1}+c_{22} N_{2}+\cdots+c_{2 n} N_{n}=b_{2} \\
\vdots \\
c_{n 1} N_{1}+c_{n 2} N_{2}+\cdots+c_{n n} N_{n}=b_{n}
\end{array}\right.
$$

Lemma 2.1. Under (2.2), for any given initial value $\mathrm{N}(\xi) \in \Phi$, model (2.1) has a unique global solution $\mathrm{N}(\mathrm{t})=$ $\left(\mathrm{N}_{1}(\mathrm{t}), \cdots, \mathrm{N}_{\mathrm{n}}(\mathrm{t})\right)^{\mathrm{T}} \in \mathrm{R}_{+}^{\mathrm{n}}$ on $\mathrm{t} \geqslant 0$ a.s.. Moreover, there exists a positive constant $\mathrm{K}_{1}$ such that

$$
\limsup _{t \rightarrow+\infty} \mathbb{E}\left(N_{i}(t)\right) \leqslant K_{1}, \quad i=1,2, \cdots, n .
$$

Proof. The proof is a slight modification of that in Bao et al. [3, Theorem 2.1 and Theorem 3.1], and hence is omitted.

Now we are in the position to give our main results.

Assumption 2.2. There is a constant $k>0$ such that

$$
\int_{\mathbb{Y}}\left[\ln \left(1+\gamma_{i}(u)\right)\right]^{2} \theta(d u)<k .
$$

Assumption 2.3. $c_{i i}>\sum_{j=1, j \neq i}^{n} c_{i j}, i=1, \cdots, n$.

Theorem 2.4. Let (2.2), Assumptions 2.2 and 2.3 hold. If $b_{i}>0$ and $f_{i}>0, i=1, \cdots, n$, then model (2.1) is $S P 2$, and at the same time, $\mu$ is ergodic with

$$
\int_{R_{+}^{n}} N \mu(d N)=\lim _{t \rightarrow+\infty} t^{-1} \int_{0}^{t} N(s) d s=\left(\frac{C_{1}}{C}, \cdots, \frac{C_{n}}{C}\right)^{\top}, \text { a.s. . }
$$

Remark 2.5. It is useful to pint out that if $b_{i}<0$ for some $i$, then the species $i$ in model (2.1) will go to extinction.

The proof of Theorem 2.4 is divided into the following three steps:

(i) First, we give some conditions under which all the species in model (2.1) are not extinctive.

(ii) Then, we establish the sufficient conditions for global attractivity of solutions of model (2.1).

(iii) Finally, we prove the existence, uniqueness and ergodicity of the invariant measure $\mu$. 


\subsection{Non-extinction}

Consider the following auxiliary equations:

$$
\begin{aligned}
d y_{i}(t)= & y_{i}\left(t^{-}\right)\left(r_{i}-c_{i i} y_{i}\left(t^{-}\right)\right) d t+\sigma_{i} y_{i}\left(t^{-}\right) d W_{i}(t) \\
& +\int_{\mathbb{Y}} y_{i}\left(t^{-}\right) \gamma_{i}(u) \tilde{\Gamma}(d t, d u), \quad i=1, \cdots, n, \\
d z_{i}(t)= & z_{\mathfrak{i}}\left(t^{-}\right)\left(r_{i}-c_{i i} z_{\mathfrak{i}}\left(t^{-}\right)-\sum_{j=1, j \neq i}^{n} c_{i j} y_{j}\left(t^{-}-\tau_{i j}\right)\right) d t \\
& +\sigma_{i} z_{\mathfrak{i}}\left(t^{-}\right) d W_{i}(t)+\int_{\mathbb{Y}} z_{\mathfrak{i}}\left(t^{-}\right) \gamma_{\mathfrak{i}}(u) \tilde{\Gamma}(d t, d u), \quad i=1, \cdots, n,
\end{aligned}
$$

where

$$
y_{\mathfrak{i}}(\xi)=z_{\mathfrak{i}}(\xi)=\mathrm{N}_{\mathfrak{i}}(\xi), \quad \xi \in[-\tau, 0], \quad \mathfrak{i}=1,2, \cdots, \mathrm{n} .
$$

Under (2.2), Bao et al. [3, Lemma 4.2] have shown that the solution of model (2.5) can be explicitly expressed as:

$$
y_{i}(t)=\frac{\exp \left\{b_{i} t+\sigma_{i} W_{i}(t)+m_{i}(t)\right\}}{y_{i}^{-1}(0)+c_{i i} \int_{0}^{t} \exp \left\{b_{i} s+\sigma_{i} W_{i}(s)+m_{i}(s)\right\} d s}, \quad i=1, \cdots, n .
$$

Similarly, the solution of model (2.6) is:

$$
z_{i}(t)=\frac{\exp \left\{b_{i} t-\sum_{j=1, j \neq i}^{n} c_{i j} \int_{0}^{t} y_{j}\left(s-\tau_{i j}\right) d s+\sigma_{i} W_{i}(t)+m_{i}(t)\right\}}{z_{i}^{-1}(0)+c_{i i} \int_{0}^{t} \exp \left\{b_{i} s-\sum_{j=1, j \neq i}^{n} c_{i j} \int_{0}^{s} y_{j}\left(u-\tau_{i j}\right) d u+\sigma_{i} W_{i}(s)+m_{i}(s)\right\} d s}, i=1, \cdots, n .
$$

Lemma 2.6 ([26]). For model (2.5), let Assumption 2.2 and (2.2) hold. If $\mathrm{b}_{\mathrm{i}}>0$, then

$$
\lim _{t \rightarrow+\infty} t^{-1} \ln y_{i}(t)=0, \lim _{t \rightarrow+\infty} t^{-1} \int_{0}^{t} y_{i}(s) d s=b_{i} / c_{i i}, \text { a.s., } i=1, \ldots, n .
$$

Now we are in the position to study the non-extinction of model (2.1).

Lemma 2.7 ([20]). Let $M(t), t \geqslant 0$, be a local martingale vanishing at time zero. If $\lim _{t \rightarrow+\infty} \rho_{M}(t)<+\infty$, then

$$
\lim _{t \rightarrow+\infty} \frac{M(t)}{t}=0 \text { a.s., }
$$

where

$$
\rho_{M}(t)=\int_{0}^{t} \frac{d\langle M, M\rangle(s)}{(1+s)^{2}}, \quad t \geqslant 0,
$$

and $\langle M, M\rangle(t)$ is Meyer's angle bracket process (see, e.g., $[1,13])$.

Theorem 2.8. Let Assumption 2.2 and (2.2) hold. If $\mathrm{b}_{\mathrm{i}}>0$ and $\mathrm{f}_{\mathrm{i}}>0, i=1, \cdots, \mathrm{n}$, then

$$
\lim _{t \rightarrow+\infty} t^{-1} \int_{0}^{t} N_{i}(s) d s=C_{i} / C, \text { a.s. } i=1, \cdots, n .
$$

Proof. An application of Itô's formula (see, e.g., [13]) to (2.1) gives

$$
\begin{aligned}
t^{-1} \ln N_{i}(t)-t^{-1} \ln N_{i}(0)= & b_{i}-c_{i i} t^{-1} \int_{0}^{t} N_{i}(s) d s-\sum_{j=1, j \neq i}^{n} c_{i j} t^{-1} \int_{0}^{t} N_{j}\left(s-\tau_{i j}\right) d s \\
& +\sigma_{i} t^{-1} W_{i}(t)+t^{-1} \int_{0}^{t} \int_{\mathbb{Y}} \ln \left(1+\gamma_{i}(u)\right) \widetilde{\Gamma}(d s, d u)
\end{aligned}
$$




$$
\begin{aligned}
= & b_{i}-\left[c_{i i} t^{-1} \int_{0}^{t} N_{i}(s) d s+\sum_{j=1, j \neq i}^{n} c_{i j} t^{-1} \int_{0}^{t} N_{j}(s) d s\right] \\
& +\sum_{j=1, j \neq i}^{n} c_{i j} t^{-1}\left[\int_{t-\tau_{i j}}^{t} N_{j}(s) d s-\int_{-\tau_{i j}}^{0} N_{j}(s) d s\right] \\
& +\sigma_{i} t^{-1} W_{i}(t)+t^{-1} m_{i}(t) .
\end{aligned}
$$

Since $b_{i}>0$, by (2.8), we can see that for $i, j=1, \cdots, n, j \neq i$,

$$
\lim _{t \rightarrow+\infty} t^{-1} \int_{t-\tau_{i j}}^{t} y_{j}(s) d s=\lim _{t \rightarrow+\infty}\left(t^{-1} \int_{0}^{t} y_{j}(s) d s-t^{-1} \int_{0}^{t-\tau_{i j}} y_{j}(s) d s\right)=0 .
$$

In view of the comparison theorem for SDEs with jumps ([33]), one can see that for $t \geqslant-\tau$,

$$
z_{\mathfrak{i}}(t) \leqslant N_{i}(t) \leqslant y_{i}(t) \text { a.s. } i=1, \cdots, n .
$$

It follows that

$$
\lim _{t \rightarrow+\infty} t^{-1} \int_{t-\tau_{i j}}^{t} N_{j}(s) d s=0, \quad i, j=1, \cdots, n, j \neq i
$$

Therefore

$$
\lim _{t \rightarrow+\infty} t^{-1}\left[\int_{t-\tau_{i j}}^{t} N_{j}(s) d s-\int_{-\tau_{i j}}^{0} N_{j}(s) d s\right]=0, \quad i, j=1, \cdots, n, j \neq i .
$$

Note that Assumption 2.2 and (2.2) hold, it then follows from Lemma 2.7 that

$$
\lim _{t \rightarrow+\infty} t^{-1} W_{i}(t)=0, \quad \lim _{t \rightarrow+\infty} t^{-1} m_{i}(t)=0, \text { a.s. } .
$$

Thus, to complete the proof, we only need to show that

$$
\lim _{t \rightarrow+\infty} t^{-1} \ln N_{i}(t)=0 \text { a.s. } i=1, \cdots, n \text {. }
$$

Thanks to (2.8) and (2.11),

$$
\liminf _{t \rightarrow+\infty} t^{-1} \ln z_{i}(t) \leqslant \liminf _{t \rightarrow+\infty} t^{-1} \ln N_{i}(t) \leqslant \limsup _{t \rightarrow+\infty} t^{-1} \ln N_{i}(t) \leqslant \limsup _{t \rightarrow+\infty} t^{-1} y_{i}(t)=0 .
$$

Consequently, we only need to prove that

$$
\liminf _{t \rightarrow+\infty} t^{-1} \ln z_{\mathfrak{i}}(t) \geqslant 0 \text { a.s. } i=1, \cdots, n .
$$

By virtue of (2.8) and (2.10), we can observe that for $i, j=1, \cdots, n, j \neq i$,

$$
\lim _{t \rightarrow+\infty} t^{-1} \int_{0}^{t} y_{j}\left(s-\tau_{i j}\right) d s=\lim _{t \rightarrow+\infty} t^{-1}\left(\int_{0}^{t} y_{j}(s) d s-\int_{t-\tau_{i j}}^{t} y_{j}(s) d s+\int_{-\tau_{i j}}^{0} y_{j}(s) d s\right)=b_{j} / c_{j j}, \text { a.s. . }
$$

Hence by (2.12), for arbitrary given $\varepsilon>0$, there exists a $T=T(\omega)$ such that for $t \geqslant T, i, j=1, \cdots, n, j \neq i$,

$$
b_{j} / c_{j j}-\varepsilon \leqslant t^{-1} \int_{0}^{t} y_{j}\left(s-\tau_{i j}\right) d s \leqslant b_{j} / c_{j j}+\varepsilon,
$$

and

$$
-\varepsilon \leqslant t^{-1} \sigma_{i} W_{i}(t)+t^{-1} m_{i}(t) \leqslant \varepsilon .
$$


When these inequalities are used in (2.7), one can see that

$$
\begin{aligned}
\frac{1}{z_{i}(t)}= & \exp \left\{-b_{i} t+\sum_{j=1, j \neq i}^{n} c_{i j} \int_{0}^{t} y_{j}\left(s-\tau_{i j}\right) d s-\sigma_{i} W_{i}(t)-m_{i}(t)\right\} \\
& \times\left\{z_{i}^{-1}(0)+c_{i i} \int_{0}^{t} \exp \left\{b_{i} s-\sum_{j=1, j \neq i}^{n} c_{i j} \int_{0}^{s} y_{j}\left(u-\tau_{i j}\right) d u+\sigma_{i} W_{i}(s)+m_{i}(s)\right\} d s\right\} \\
= & \exp \left\{-b_{i} t+\sum_{j=1, j \neq i}^{n} c_{i j} \int_{0}^{t} y_{j}\left(s-\tau_{i j}\right) d s-\sigma_{i} W_{i}(t)-m_{i}(t)\right\} \\
& \times\left\{z_{i}^{-1}(0)+c_{i i} \int_{0}^{T} \exp \left[b_{i} s-\sum_{j=1, j \neq i}^{n} c_{i j} \int_{0}^{s} y_{j}\left(u-\tau_{i j}\right) d u+\sigma_{i} W_{i}(s)+m_{i}(s)\right] d s\right. \\
& \left.+c_{i i} \int_{T}^{t} \exp \left[b_{i} s-\sum_{j=1, j \neq i}^{n} c_{i j} \int_{0}^{s} y_{j}\left(u-\tau_{i j}\right) d u+\sigma_{i} W_{i}(s)+m_{i}(s)\right] d s\right\} \\
\leqslant & \exp \left\{t\left[-b_{i}+\sum_{j=1, j \neq i}^{n} c_{i j}\left(\frac{b_{j}}{c_{j j}}+\varepsilon\right)+\varepsilon\right]\right\} \\
& \times\left\{z_{i}^{-1}(0)+k_{i}+c_{i i} \int_{T}^{t} \exp \left\{s\left[b_{i}-\sum_{j=1, j \neq i}^{n} c_{i j}\left(\frac{b_{j}}{c_{j j}}-\varepsilon\right)+\varepsilon\right]\right\} d s\right\} \\
= & \exp \left\{t\left[-f_{i}+\left(1+\sum_{j=1, j \neq i}^{n} c_{i j}\right) \varepsilon\right]\right\} \\
& \times\left\{z_{i}^{-1}(0)+K_{i}+c_{i i} \int_{T}^{t} \exp \left\{s\left[f_{i}+\left(1+\sum_{j=1, j \neq i}^{n} c_{i j}\right) \varepsilon\right]\right\} d s\right\},
\end{aligned}
$$

where $K_{i}>0$ is a constant. Note that $f_{i}>0$, hence for sufficiently large $t$, one can derive that

$$
z_{i}^{-1}(0)+K_{i} \leqslant c_{i i} \int_{T}^{t} \exp \left\{s\left[f_{i}+\left(1+\sum_{j=1, j \neq i}^{n} c_{i j}\right) \varepsilon\right]\right\} d s .
$$

That is to say, for sufficiently large $t$,

$$
\begin{aligned}
\frac{1}{z_{i}(t)} \leqslant & \exp \left\{t\left[-f_{i}+\left(1+\sum_{j=1, j \neq i}^{n} c_{i j}\right) \varepsilon\right]\right\} \\
& \times 2 c_{i i} \int_{T}^{t} \exp \left\{s\left[f_{i}+\left(1+\sum_{j=1, j \neq i}^{n} c_{i j}\right) \varepsilon\right]\right\} d s \\
= & \frac{2 c_{i i}}{f_{i}+\left(1+\sum_{j=1, j \neq i}^{n} c_{i j}\right) \varepsilon} \exp \left\{t\left[-f_{i}+\left(1+\sum_{j=1, j \neq i}^{n} c_{i j}\right) \varepsilon\right]\right\} \\
& \times \exp \left\{\left[f_{i}+\left(1+\sum_{j=1, j \neq i}^{n} c_{i j}\right) \varepsilon\right](t-T)\right\} .
\end{aligned}
$$

Consequently

$$
\begin{aligned}
t^{-1} \ln z_{i}(t) \geqslant & t^{-1} \ln \frac{f_{i}+\left(1+\sum_{j=1, j \neq i}^{n} c_{i j}\right) \varepsilon}{2 c_{i i}}-2\left(1+\sum_{j=1, j \neq i}^{n} c_{i j}\right) \varepsilon \\
& +\left[f_{i}+\left(1+\sum_{j=1, j \neq i}^{n} c_{i j}\right) \varepsilon\right] T / t .
\end{aligned}
$$


Letting $t \rightarrow+\infty$ and then making use of the arbitrariness of $\varepsilon$, one can get the desired assertion (2.13).

\subsection{Global attractivity}

Theorem 2.9. If Assumption 2.3 holds, then model (2.1) is globally attractive, i.e.,

$$
\lim _{t \rightarrow+\infty} \sum_{i=1}^{n} \mathbb{E}\left|N_{i}(\phi ; t)-N_{i}(\varphi ; t)\right|=0 \text {, a.s., }
$$

where $\mathrm{N}(\phi ; \mathrm{t})=\left(\mathrm{N}_{1}(\phi ; \mathrm{t}), \cdots, \mathrm{N}_{\mathrm{n}}(\phi ; \mathrm{t})\right)^{\top}$ and $\mathrm{N}(\varphi ; \mathrm{t})=\left(\mathrm{N}_{1}(\varphi ; \mathrm{t}), \cdots, \mathrm{N}_{\mathrm{n}}(\varphi ; \mathrm{t})\right)^{\top}$ are two solutions of model (2.1) with initial data $\phi(\xi) \in \Phi$ and $\varphi(\xi) \in \Phi$, respectively.

Proof. Let $\beta_{i}$ represent the cofactor of the $i$-th diagonal element of $L_{C}$, where

$$
\mathrm{L}_{\mathrm{C}}=\left(\begin{array}{cccc}
\sum_{j=2}^{n} \mathrm{c}_{1 j} & -\mathrm{c}_{12} & \cdots & -\mathrm{c}_{1 n} \\
-\mathrm{c}_{21} & \sum_{j=1, j \neq 2}^{n} c_{2 j} & \cdots & -c_{2 n} \\
\cdots & \cdots & \cdots & \cdots \\
-c_{n 1} & -c_{n 2} & \cdots & \sum_{j=1}^{n-1} c_{n j}
\end{array}\right) .
$$

By Kirchhoff's Matrix Tree Theorem (see e.g., [32]), we have $\beta_{i}>0, i=1, \cdots, n$.

Define (the following $V$ function is motivated by [25])

$$
V(t)=\sum_{i=1}^{n} \beta_{i}\left|\ln N_{i}(\phi ; t)-\ln N_{i}(\varphi ; t)\right|+\sum_{i=1}^{n} \sum_{j=1, j \neq i}^{n} \beta_{i} c_{i j} \int_{t-\tau_{i j}}^{t}\left|N_{j}(\phi ; s)-N_{j}(\varphi ; s)\right| d s .
$$

Making use of Itô's formula, we have

$$
\begin{aligned}
d^{+} V(t)= & \sum_{i=1}^{n} \beta_{i} \operatorname{sgn}\left(N_{i}(\phi ; t)-N_{i}(\varphi ; t)\right) d\left(\ln N_{i}(\phi ; t)-\ln N_{i}(\varphi ; t)\right) \\
& +\sum_{i=1}^{n} \sum_{j=1, j \neq i}^{n} \beta_{i} c_{i j}\left|N_{j}(\phi ; t)-N_{j}(\varphi ; t)\right| d t-\sum_{i=1}^{n} \sum_{j=1, j \neq i}^{n} \beta_{i} c_{i j}\left|N_{j}\left(\phi ; t-\tau_{i j}\right)-N_{j}\left(\varphi ; t-\tau_{i j}\right)\right| d t \\
= & \sum_{i=1}^{n} \beta_{i} \operatorname{sgn}\left(N_{i}(\phi ; t)-N_{i}(\varphi ; t)\right)\left[-c_{i i}\left(N_{i}(\phi ; t)-N_{i}(\varphi ; t)\right)\right. \\
& \left.-\sum_{j=1, j \neq i}^{n} c_{i j}\left(N_{j}\left(\phi ; t-\tau_{i j}\right)-N_{j}\left(\varphi ; t-\tau_{i j}\right)\right)\right] d t \\
& +\sum_{i=1}^{n} \sum_{j=1, j \neq i}^{n} \beta_{i} c_{i j}\left|N_{j}(\phi ; t)-N_{j}(\varphi ; t)\right| d t-\sum_{i=1}^{n} \sum_{j=1, j \neq i}^{n} \beta_{i} c_{i j}\left|N_{j}\left(\phi ; t-\tau_{i j}\right)-N_{j}\left(\varphi ; t-\tau_{i j}\right)\right| d t \\
\leqslant & -\sum_{i=1}^{n} \beta_{i} c_{i i}\left|N_{i}(\phi ; t)-N_{i}(\varphi ; t)\right| d t+\sum_{i=1}^{n} \sum_{j=1, j \neq i}^{n} \beta_{i} c_{i j}\left|N_{j}\left(\phi ; t-\tau_{i j}\right)-N_{j}\left(\varphi ; t-\tau_{i j}\right)\right| d t \\
& +\sum_{i=1}^{n} \sum_{j=1, j \neq i}^{n} \beta_{i} c_{i j}\left|N_{j}(\phi ; t)-N_{j}(\varphi ; t)\right| d t-\sum_{i=1}^{n} \sum_{j=1, j \neq i}^{n} \beta_{i} c_{i j}\left|N_{j}\left(\phi ; t-\tau_{i j}\right)-N_{j}\left(\varphi ; t-\tau_{i j}\right)\right| d t \\
= & -\sum_{i=1}^{n} \beta_{i} c_{i i}\left|N_{i}(\phi ; t)-N_{i}(\varphi ; t)\right| d t+\sum_{i=1}^{n} \sum_{j=1, j \neq i}^{n} \beta_{i} c_{i j}\left|N_{j}(\phi ; t)-N_{j}(\varphi ; t)\right| d t .
\end{aligned}
$$


In view of [18, Theorem 2.3],

$$
\sum_{i=1}^{n} \sum_{j=1, j \neq i}^{n} \beta_{i} c_{i j}\left|N_{j}(\phi ; t)-N_{j}(\varphi ; t)\right|=\sum_{i=1}^{n} \sum_{j=1, j \neq i}^{n} \beta_{i} c_{i j}\left|N_{i}(\phi ; t)-N_{i}(\varphi ; t)\right| .
$$

Consequently,

$$
\mathbb{E}(V(t)) \leqslant V(0)-\sum_{i=1}^{n} \beta_{i}\left[c_{i i}-\sum_{j=1, j \neq i}^{n} c_{i j}\right] \int_{0}^{t} \mathbb{E}\left|N_{i}(\phi ; s)-N_{i}(\varphi ; s)\right| d s .
$$

It then follows from $\mathbb{E}(\mathrm{V}(\mathrm{t})) \geqslant 0$ that

$$
\sum_{i=1}^{n} \beta_{i}\left[c_{i i}-\sum_{j=1, j \neq i}^{n} c_{i j}\right] \int_{0}^{t} \mathbb{E}\left|N_{i}(\phi ; s)-N_{i}(\varphi ; s)\right| d s \leqslant V(0)<\infty .
$$

That is to say,

$$
\mathbb{E}\left|N_{i}(\phi ; t)-N_{i}(\varphi ; t)\right| \in L^{1}[0, \infty), \quad i=1, \cdots, n .
$$

On the other hand, by (2.1),

$$
\mathbb{E}\left(N_{i}(t)\right)=N_{i}(0)+\int_{0}^{t}\left[\mathbb{E}\left(N_{i}(s)\right) r_{i}-c_{i i} \mathbb{E}\left(N_{i}(s)\right)^{2}-\sum_{j=1, j \neq i}^{n} c_{i j} \mathbb{E}\left(N_{i}(s) N_{j}\left(s-\tau_{i j}\right)\right)\right] d s
$$

It follows that $\mathbb{E}\left(\mathrm{N}_{\mathfrak{i}}(\mathrm{t})\right)$ is continuously differentiable with respect to $t$. According to (2.3),

$$
\frac{d \mathbb{E}\left(N_{i}(t)\right)}{d t}=\mathbb{E}\left(N_{i}(t)\right) r_{i}-c_{i i} \mathbb{E}\left(N_{i}(t)\right)^{2}-\sum_{j=1, j \neq i}^{n} c_{i j} \mathbb{E}\left(N_{i}(t) N_{j}\left(t-\tau_{i j}\right)\right) \leqslant \mathbb{E}\left(N_{i}(t)\right) r_{i} \leqslant r_{i} K_{1} .
$$

Thereby, $\mathbb{E}\left(N_{i}(t)\right)$ is uniformly continuous with respect to $t$. Then the required assertion (2.14) follows from Barbalat's results [4].

\subsection{Proof of Theorem 2.4}

Proof of Theorem 2.4. The proof is motivated by [23, 25, 28]. To begin with, let us prove the existence and uniqueness of the measure $\mu$. Let $p(t, \phi, \cdot)$ be the transition probability of $N(t)$, and let $P(t, \phi, B)$ be the probability of $N(t) \in B$. It then follows from (2.3) and the Chebyshev inequality that the family of transition probability $\{\mathrm{p}(\mathrm{t}, \phi, \cdot)\}$ is tight.

Let $\Lambda(\Phi)$ stand for all the probability measures defined on $\Phi$. For any two measures $P_{1}, P_{2} \in \Lambda$, define the following Kantorovich metric ([5])

$$
d_{H}\left(P_{1}, P_{2}\right)=\sup _{h \in H}\left|\int_{R_{+}^{n}} h(N) P_{1}(d N)-\int_{R_{+}^{n}} h(N) P_{2}(d N)\right|,
$$

where

$$
H=\{h: \Phi \rightarrow R|| h(x)-h(y)|\leqslant\|x-y\|,| h(\cdot) \mid \leqslant 1\} .
$$

For any $h \in H$ and $t, s>0$, we have

$$
\begin{aligned}
|\mathbb{E h}(\mathrm{N}(\phi ; \mathrm{t}+\mathrm{s}))-\mathbb{E h}(\mathrm{N}(\phi ; \mathrm{t}))| & =\left|\mathbb{E}\left[\mathbb{E}\left(\mathrm{h}(\mathrm{N}(\phi ; \mathrm{t}+\mathrm{s})) \mid \mathcal{F}_{s}\right)\right]-\mathbb{E h}(\mathrm{N}(\phi ; \mathrm{t}))\right| \\
& =\left|\int_{R_{+}^{n}} \operatorname{Eh}(\mathrm{N}(\varphi ; \mathrm{t})) \mathrm{p}(\mathrm{s}, \phi, \mathrm{d} \varphi)-\mathbb{E h}(\mathrm{N}(\phi ; \mathrm{t}))\right| \\
& \leqslant \int_{R_{+}^{n}}|\mathbb{E h}(\mathrm{N}(\varphi ; \mathrm{t}))-\mathbb{E h}(\mathrm{N}(\phi ; \mathrm{t}))| \mathrm{p}(\mathrm{s}, \phi, \mathrm{d} \varphi) .
\end{aligned}
$$


Thanks to (2.14), there is a $T>0$ such that for $t \geqslant T$,

$$
\sup _{h \in H}|\operatorname{Eh}(N(\varphi ; t))-\mathbb{E h}(N(\phi ; t))| \leqslant \varepsilon .
$$

Substituting this inequality into (2.15), and then using the tightness of $\{\mathrm{p}(\mathrm{t}, \phi, \cdot)\}$, we obtain

$$
|\mathbb{E h}(N(\phi ; t+s))-\mathbb{E h}(N(\phi ; t))| \leqslant \varepsilon
$$

It then follows from the arbitrariness of $h$ that

$$
\sup _{h \in H}|\mathbb{E h}(N(\phi ; t+s))-\mathbb{E h}(N(\phi ; t))| \leqslant \varepsilon .
$$

That is to say,

$$
\mathrm{d}_{\mathrm{H}}(\mathrm{p}(\mathrm{t}+\mathrm{s}, \phi, \cdot), \mathrm{p}(\mathrm{t}, \phi, \cdot)) \leqslant \varepsilon, \quad \forall \mathrm{t} \geqslant \mathrm{T}, \quad \mathrm{s}>0 .
$$

Consequently, $\{p(t, \phi, \cdot): t \geqslant 0\}$ is Cauchy in the space $\Lambda(\Phi)$. It follows that there exists a unique measure $\mu(\cdot) \in \Lambda(\Phi)$ such that

$$
\lim _{t \rightarrow+\infty} d_{H}(p(t, k, \cdot), \mu(\cdot))=0,
$$

where $\kappa=\kappa(\xi) \equiv(0.1, \cdots, 0.1)^{\top}, \xi \in[-\tau, 0]$. But from (2.14), we have

$$
\lim _{t \rightarrow+\infty} d_{H}(p(t, \phi, \cdot), p(t, k, \cdot))=0 .
$$

Therefore

$$
\lim _{t \rightarrow+\infty} d_{H}(p(t, \phi, \cdot), \mu(\cdot)) \leqslant \lim _{t \rightarrow+\infty} d_{H}(p(t, \phi, \cdot), p(t, k, \cdot))+\lim _{t \rightarrow+\infty} d_{H}(p(t, k, \cdot), \mu(\cdot))=0 .
$$

This completes the proof of the existence and uniqueness of $\mu$. At the same time, by $(2.9), \mu\left(\Lambda_{0}\right)=0$.

Now we are in the position to prove the ergodicity of $\mu$. In fact, the distribution of $N(t)$ converges to $\mu$ as $t \rightarrow+\infty$. In view of [9, Corollary 3.4.3], we can see that $\mu(\cdot)$ is strong mixing. It then follows from [9, Theorem 3.2.6] that $\mu(\cdot)$ is ergodic. By virtue of (3.3.2) in [9], one can observe that

$$
\lim _{t \rightarrow+\infty} t^{-1} \int_{0}^{t} N(s) d s=\int_{R_{+}^{n}} N \mu(d N) .
$$

This, together with (2.9), means (2.4).

Remark 2.10. When Theorem 2.4 is applied to the logistic model (1.4), we can obtain that if $r-\frac{\sigma^{2}}{2}>0$, the distribution of $N(t)$ converges to a unique measure $v$ which is ergodic:

$$
\lim _{t \rightarrow+\infty} t^{-1} \int_{0}^{t} N(s) d s=\int_{R_{+}} N v(d N)=K\left(r-\frac{\sigma^{2}}{2}\right)
$$

Therefore,

$$
\lambda(v)=\int_{R_{+}} \lambda(N) v(d N)=\int_{R_{+}}\left(r-\frac{N}{K}-\frac{1}{2} \sigma^{2}\right) v(d N)=r-\frac{1}{2} \sigma^{2}-\left(r-\frac{1}{2} \sigma^{2}\right)=0 .
$$

Hence we can not use the results in [35] to investigate the SP2 of model (1.4).

Under (2.2), Assumptions 2.2 and 2.3, Theorem 2.4 has established the sufficient conditions for SP2 of 
model (2.1). In the following, we shall show that these conditions are sharp when $n=2$. Consider the following model:

with initial data

$$
\left\{\begin{aligned}
\mathrm{dN}_{1}(t)= & \mathrm{N}_{1}\left(\mathrm{t}^{-}\right)\left[\mathrm{r}_{1}-\mathrm{c}_{11} \mathrm{~N}_{1}\left(\mathrm{t}^{-}\right)-\mathrm{c}_{12} \mathrm{~N}_{2}\left(\mathrm{t}^{-}-\tau_{12}\right)\right] \mathrm{dt} \\
& +\sigma_{1} \mathrm{~N}_{1}\left(\mathrm{t}^{-}\right) \mathrm{d} W_{1}(\mathrm{t})+\mathrm{N}_{1}\left(\mathrm{t}^{-}\right) \int_{\mathbb{Y}} \gamma_{1}(u) \tilde{\Gamma}(\mathrm{dt}, \mathrm{du}), \\
\mathrm{dN}_{2}(\mathrm{t})= & \mathrm{N}_{2}\left(\mathrm{t}^{-}\right)\left[\mathrm{r}_{2}-\mathrm{c}_{21} \mathrm{~N}_{1}\left(\mathrm{t}^{-}-\tau_{21}\right)-\mathrm{c}_{22} \mathrm{~N}_{2}\left(\mathrm{t}^{-}\right)\right] \mathrm{dt} \\
& +\sigma_{2} \mathrm{~N}_{2}\left(\mathrm{t}^{-}\right) d W_{2}(\mathrm{t})+\mathrm{N}_{2}\left(\mathrm{t}^{-}\right) \int_{\mathbb{Y}} \gamma_{2}(\mathrm{u}) \tilde{\Gamma}(\mathrm{dt}, \mathrm{du}),
\end{aligned}\right.
$$

$$
\left(\mathrm{N}_{1}(\xi), \mathrm{N}_{2}(\xi)\right)^{\top}=\left(\phi_{1}(\xi), \phi_{2}(\xi)\right)^{\top} \in \Phi
$$

Lemma 2.11 ([26]). For model (2.16), let (2.2) and Assumption 2.2 hold. If $\mathrm{b}_{1}>0, \mathrm{~b}_{2}>0$ and $\Delta:=\mathrm{c}_{11} \mathrm{c}_{22}-$ $\mathrm{c}_{12} \mathrm{c}_{21}>0$, then

(i) if $\mathrm{f}_{1}>0$ and $\mathrm{f}_{2}<0$, then $\mathrm{N}_{2}$ goes extinct a.s.;

(ii) if $\mathrm{f}_{1}<0$ and $\mathrm{f}_{2}>0$, then $\mathrm{N}_{1}$ goes extinct a.s.

Clearly, Assumption 2.3 means that $\Delta>0$. Moreover, it is easy to see that if $\Delta>0$, then $f_{1}<0$ and $\mathrm{f}_{2}<0$ can not hold simultaneously. Therefore, by Lemma 2.11 and Theorem 2.4, we have:

Corollary 2.12. For model (2.16), let (2.2), Assumption 2.2 and Assumption 2.3 hold. If $\mathrm{b}_{1}>0$ and $\mathrm{b}_{2}>0$, then:

(a) if $\mathrm{f}_{1}>0$ and $\mathrm{f}_{2}>0$, then model (2.16) is SP2, and at the same time, $\mu$ is ergodic with

$$
\int_{R_{+}^{2}} N \mu(d N)=\lim _{t \rightarrow+\infty} t^{-1} \int_{0}^{t} N(s) d s=\left(\frac{b_{1} c_{22}-b_{2} c_{12}}{c_{11} c_{22}-c_{12} c_{21}}, \frac{b_{2} c_{11}-b_{1} c_{21}}{c_{11} c_{22}-c_{12} c_{21}}\right)^{\top} \text {, a.s.; }
$$

(b) if $\mathrm{f}_{1}>0$ and $\mathrm{f}_{2}<0$, then $\mathrm{N}_{2}$ goes extinct a.s., and $\mathrm{N}_{1}$ is SP2 with an ergodic measure $\bar{\mu}$ :

$$
\int_{R_{+}} N_{1} \bar{\mu}\left(d N_{1}\right)=\lim _{t \rightarrow+\infty} t^{-1} \int_{0}^{t} N_{1}(s) d s=\frac{b_{1}}{c_{11}}, \text { a.s.; }
$$

(c) if $\mathrm{f}_{1}<0$ and $\mathrm{f}_{2}>0$, then $\mathrm{N}_{1}$ goes extinct a.s., and $\mathrm{N}_{2}$ is SP2 with an ergodic measure $\hat{\mu}$ :

$$
\int_{R_{+}} N_{2} \hat{\mu}\left(d N_{2}\right)=\lim _{t \rightarrow+\infty} t^{-1} \int_{0}^{t} N_{2}(s) d s=\frac{b_{2}}{c_{22}} \text {, a.s. . }
$$

\section{Numerical simulations}

Now let us work out some numerical figures to illustrate the results. For simplicity, let us consider model (2.16) with $r_{1}=0.8, r_{2}=0.5, c_{11}=0.5, c_{12}=0.4, c_{21}=0.3, c_{22}=0.4, \sigma_{2}^{2}=0.3, \mathbb{Y}=[0,+\infty)$, $\theta(\mathbb{Y})=1, \gamma_{1}(u) \equiv 0.5162, \gamma_{2}(u) \equiv 0.3504, \tau_{1}=\tau_{2}=5, N_{1}(\theta)=0.5+0.1 \sin \theta, N_{2}(\theta)=0.4-0.2 \sin \theta$. Clearly, (2.2), Assumptions 2.2 and 2.3 hold, and $b_{2}=0.3$. The only difference between Figure 1, Figure 2 and Figure 3 is that the value of $\sigma_{1}^{2}$ is different.

- In Figure 1 , we choose $\sigma_{1}^{2} / 2=0.3$, then $b_{1}=0.4$. According to (a) in Corollary 2.12, model (2.16) is SP2 and

$$
\begin{aligned}
& \lim _{t \rightarrow+\infty} t^{-1} \int_{0}^{t} N_{1}(s) d s=0.5 \\
& \lim _{t \rightarrow+\infty} t^{-1} \int_{0}^{t} N_{2}(s) d s=0.375 .
\end{aligned}
$$


See Figure 1. Figure 1 (a) is a sample path and Figure $1(b)$ is the density of the distribution at time $\mathrm{t}=5000$ ([6]).

- In Figure 2, we set $\sigma_{1}^{2} / 2=0.1$, then $b_{1}=0.6$. By virtue of $(b)$ in Corollary $2.12, \mathrm{~N}_{1}$ is SP2, $\lim _{t \rightarrow+\infty} t^{-1} \int_{0}^{t} N_{1}(s) d s=1.2$, and $N_{2}$ goes extinct, see Figure 2.

- In Figure 3, we choose $\sigma_{1}^{2} / 2=0.5$, then $b_{1}=0.2$. In view of (c) in Corollary $2.12, \mathrm{~N}_{1}$ goes extinct, $\mathrm{N}_{2}$ is SP2, and $\lim _{\mathrm{t} \rightarrow+\infty} \mathrm{t}^{-1} \int_{0}^{\mathrm{t}} \mathrm{N}_{2}(\mathrm{~s}) \mathrm{ds}=0.75$, see Figure 3 .

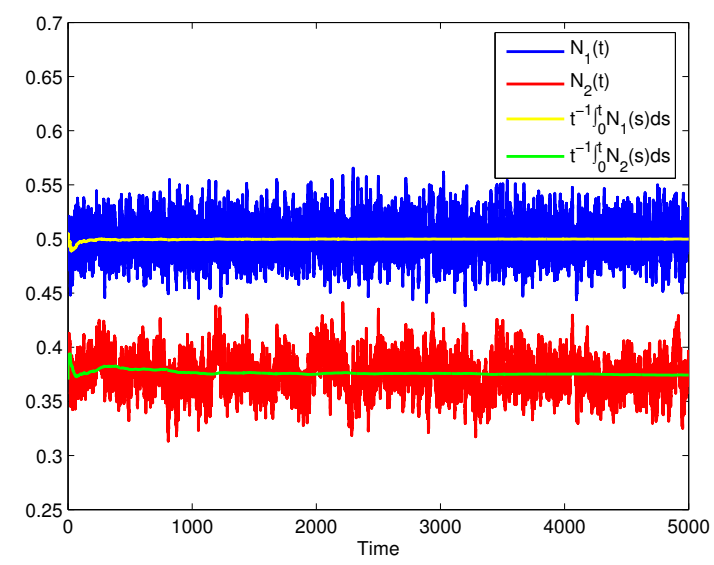

(a)

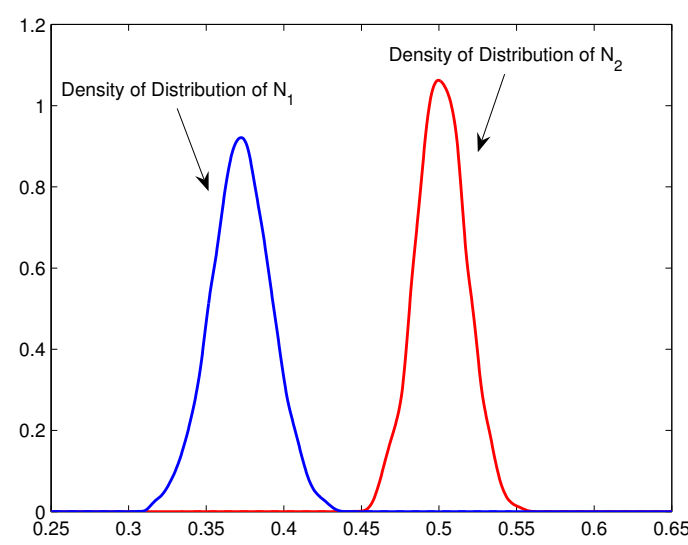

(b)

Figure 1: Model (2.16) with $r_{1}=0.8, r_{2}=0.5, c_{11}=0.5, c_{12}=0.4, c_{21}=0.3, c_{22}=0.4, \sigma_{2}^{2}=0.3, \mathbb{Y}=[0,+\infty), \theta(\mathbb{Y})=1$, $\gamma_{1}(u) \equiv 0.1, \gamma_{2}(u) \equiv 0.05, \sigma_{1}^{2} / 2=0.3, \tau_{1}=\tau_{2}=5, N_{1}(\theta)=0.5+0.1 \sin \theta, N_{2}(\theta)=0.4-0.2 \sin \theta$. (a) is a sample path; (b) is the density of the distribution at time $t=5000$.

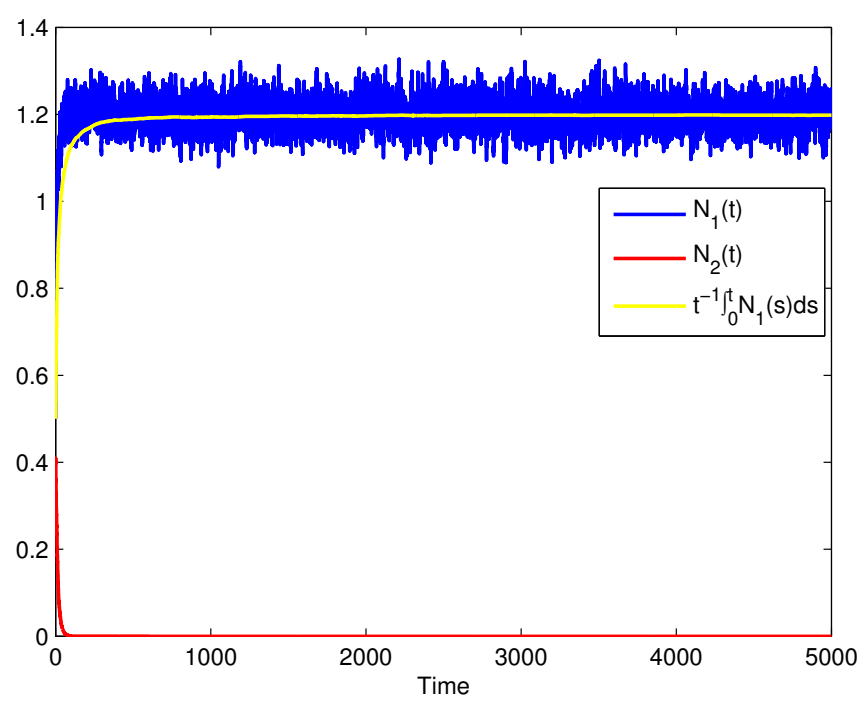

Figure 2: A sample path of model (2.16) with the same parameter values given in Figure 1 except $\sigma_{1}^{2} / 2=0.1$. 


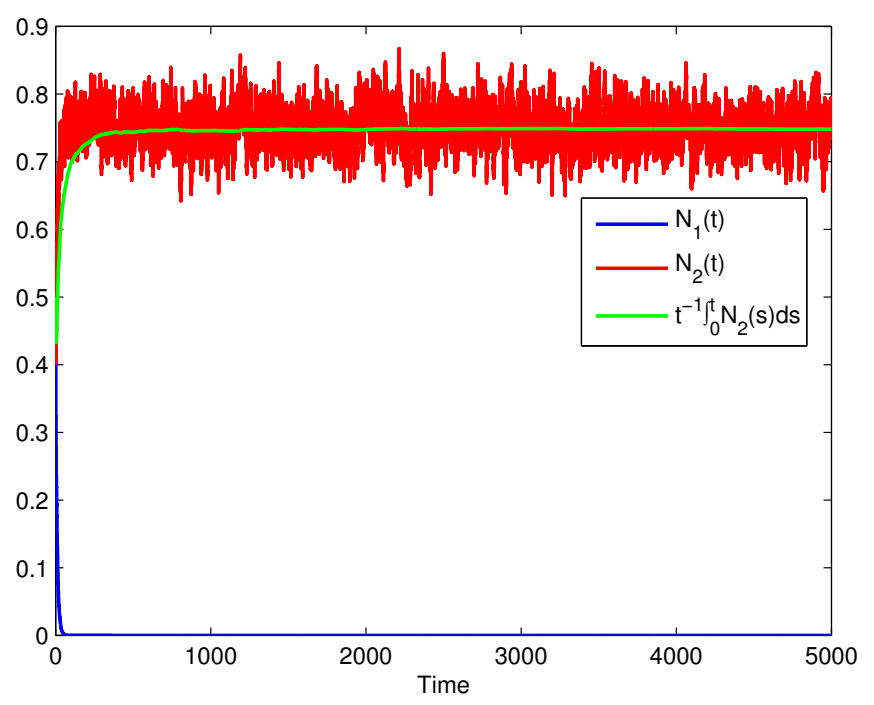

Figure 3: A sample path of model (2.16) with the same parameter values given in Figure 1 except $\sigma_{1}^{2} / 2=0.5$.

\section{Conclusions and discussions}

Permanence is one of the most interesting and important topics in ecology and epidemics. However, one widely used concept of permanence for stochastic population models, SP1, can not ensure the longterm survival of all species. Schreiber et al. [35] proposed a more appropriate concept, SP2. However, the methods in [35] can not apply to investigate most stochastic population models. In this paper, we use an asymptotic approach to investigate the SP2. Sufficient criteria for the SP2 of a stochastic delay competition model with Lévy jumps are established. We also show that these criteria are sharp when $n=2$.

Our results show that the stochastic perturbations play a key role in determining the SP2 of the models. To see this more clearly, let us consider model (2.16). Corollary 2.12 shows that the permanence or not of species $i$ depends only on the sign of $f_{i}, i=1,2$, where

$$
\begin{aligned}
& f_{1}=b_{1}-\frac{c_{12}}{c_{22}} b_{2}, f_{2}=b_{2}-\frac{c_{21}}{c_{11}} b_{1}, \\
& b_{i}=r_{i}-0.5 \sigma_{i}^{2}-\int_{\mathbb{Y}}\left[\gamma_{i}(u)-\ln \left(1+\gamma_{i}(u)\right)\right] \theta(d u) .
\end{aligned}
$$

Firstly, let us consider the effects of white noises. Clearly,

$$
\frac{\partial f_{1}}{\partial\left(\sigma_{1}^{2}\right)}<0, \frac{\partial f_{1}}{\partial\left(\sigma_{2}^{2}\right)}>0, \frac{\partial f_{2}}{\partial\left(\sigma_{1}^{2}\right)}>0, \frac{\partial f_{2}}{\partial\left(\sigma_{2}^{2}\right)}<0 .
$$

Therefore, with the increasing of $\sigma_{i}^{2}$, the species $i$ tends to go extinct while the species $j$ tends to be permanent, $i, j=1,2, i \neq j$. That is to say, the white noise of a species is harmful for the permanence of this species and is favorable for the permanence of its competitor.

Now let us consider the effect of Lévy noises. For simplicity, we let $\gamma_{i}(u)$ be a constant, $\kappa_{i}$, hence

$$
b_{i}=r_{i}-0.5 \sigma_{i}^{2}-\left(\kappa_{i}-\ln \left(1+\kappa_{i}\right)\right), \quad i=1,2 .
$$

Not that

$$
a-\ln (1+a)=a+1-1-\ln (1+a) \geqslant 0, \quad a>-1,
$$

thus

$$
b_{i} \leqslant r_{i}-0.5 \sigma_{i}^{2}, \quad i=1,2 .
$$


Therefore the Lévy noise of a species is harmful for the permanence of this species and is favorable for the permanence of its competitor.

It is useful to point out that the method used in this paper can also be used to study other stochastic population models with/without time delay and/or Lévy noises. For example, consider the following delay stochastic predator-prey model with delay ([26]):

$$
\left\{\begin{aligned}
\mathrm{dN}_{1}(t)= & \mathrm{N}_{1}\left(\mathrm{t}^{-}\right)\left[\mathrm{r}_{1}-\mathrm{c}_{11} \mathrm{~N}_{1}\left(\mathrm{t}^{-}\right)-\mathrm{c}_{12} \mathrm{~N}_{2}\left(\mathrm{t}^{-}-\tau_{12}\right)\right] d t \\
& +\sigma_{1} \mathrm{~N}_{1}\left(\mathrm{t}^{-}\right) d W_{1}(\mathrm{t})+\mathrm{N}_{1}\left(\mathrm{t}^{-}\right) \int_{\mathbb{Y}} \gamma_{1}(u) \tilde{\Gamma}(\mathrm{dt}, \mathrm{du}), \\
\mathrm{dN}_{2}(\mathrm{t})= & \mathrm{N}_{2}\left(\mathrm{t}^{-}\right)\left[\mathrm{r}_{2}-\mathrm{c}_{21} \mathrm{~N}_{1}\left(\mathrm{t}^{-}-\tau_{21}\right)-\mathrm{c}_{22} \mathrm{~N}_{2}\left(\mathrm{t}^{-}\right)\right] \mathrm{dt} \\
& +\sigma_{2} \mathrm{~N}_{2}\left(\mathrm{t}^{-}\right) d W_{2}(\mathrm{t})+\mathrm{N}_{2}\left(\mathrm{t}^{-}\right) \int_{\mathbb{Y}} \gamma_{2}(\mathrm{u}) \tilde{\Gamma}(\mathrm{dt}, \mathrm{du}),
\end{aligned}\right.
$$

with initial data

$$
\left(\mathrm{N}_{1}(\xi), \mathrm{N}_{2}(\xi)\right)^{\top}=\left(\phi_{1}(\xi), \phi_{2}(\xi)\right)^{\top} \in \Phi,
$$

where $r_{2}<0, c_{21}<0$. For model (4.1), Liu et al. [26] have shown that if $b_{1}<0$, then both species 1 and 2 go extinct a.s.; if $b_{1}>0$ and $f_{2}<0$, then species 2 goes extinct a.s. Clearly, $f_{2}>0$ means $b_{1}>0$. According to the steps below Theorem 2.4, similar to the proof of Theorem 2.4, we can show that for model (4.1),

- if $f_{2}>0$, then model (4.1) is SP2, and at the same time, $\mu$ is ergodic with

$$
\int_{R_{+}^{2}} N \mu(d N)=\lim _{t \rightarrow+\infty} t^{-1} \int_{0}^{t} N(s) d s=\left(\frac{b_{1} c_{22}-b_{2} c_{12}}{c_{11} c_{22}-c_{12} c_{21}}, \frac{b_{2} c_{11}-b_{1} c_{21}}{c_{11} c_{22}-c_{12} c_{21}}\right)^{\top}, \text { a.s.; }
$$

- if $b_{1}>0$ and $f_{2}<0$, then $N_{2}$ goes extinct a.s., and $N_{1}$ is SP2 with an ergodic measure $\tilde{\mu}$ :

$$
\int_{R_{+}} N_{1} \widetilde{\mu}\left(d N_{1}\right)=\lim _{t \rightarrow+\infty} t^{-1} \int_{0}^{t} N_{1}(s) d s=\frac{b_{1}}{c_{11}} \text {, a.s.; }
$$

- if $\mathrm{b}_{1}<0$, then both $\mathrm{N}_{1}$ and $\mathrm{N}_{2}$ go extinct a.s.

Some interesting questions deserve further investigation. In this paper, we consider the stochastic perturbations. The fractional derivative is another tool to show long term effects, and the population models often are discrete, then it is interesting to consider discrete fractional population models (see e.g. $[40,41])$. It is also interesting to consider the necessity of conditions in Theorem 2.4. Another problem of interest is to consider some more realistic but complex models (see, e.g., $[2,19,43,44]$ ), or other stochastic delay population models, for example, mutualism models (see, e.g., [45]), food chain models (see, e.g., [21]). All these questions are left for future study.

\section{Acknowledgment}

The authors thank Prof. Dumitru Baleanu and the reviewers for those valuable comments. The author also thank the National Natural Science Foundation of P. R. China (grant numbers 11301207 and 11401244), Project Fund from China Postdoctoral Science Foundation (grant numbers 2015M571349, 2016T90236), Jiangsu Province "333 High-Level Personnel Training Project”, Overseas Training Program for Outstanding Young College Teachers and Principals in Jiangsu Province, Qinglan Project of Jingsu Province.

\section{References}

[1] D. Applebaum, Lévy processes and stochastic calculus, Second edition, Cambridge Studies in Advanced Mathematics, Cambridge University Press, Cambridge, (2009). 2.7

[2] C.-Z. Bai, Multiplicity of solutions for a class of non-local elliptic operators systems, Bull. Korean Math. Soc., 54 (2017), 715-729.4 
[3] J.-H. Bao, X.-R. Mao, G. Yin, C.-G. Yuan, Competitive Lotka-Volterra population dynamics with jumps, Nonlinear Anal., 74 (2011), 6601-6616. 1, 2, 2, 2, 2.1

[4] I. Barbălat, Systémes d'équations différentielles d'oscillations non linéaires, (French) Rev. Math. Pures Appl., 4 (1959), 267-270. 2.2

[5] C. A. Cabrelli, U. M. Molter, The Kantorovich metric for probability measures on the circle, J. Comput. Appl. Math., 57 (1995), 345-361. 2.3

[6] Y.-L. Cai, Y. Kang, M. Banerjee, W.-M. Wang, A stochastic SIRS epidemic model with infectious force under intervention strategies, J. Differential Equations, 259 (2015), 7463-7502. 3

[7] P. L. Chesson, Persistence of a Markovian population in a patchy environment, Z. Wahrsch. Verw. Gebiete, 66 (1984), 97-107. 1

[8] P. L. Chesson, S. Ellner, Invasibility and stochastic boundedness in monotonic competition models, J. Math. Biol., 27 (1989), 117-138. 1

[9] G. Da Prato, J. Zabczyk, Ergodicity for infinite-dimensional systems, London Mathematical Society Lecture Note Series, Cambridge University Press, Cambridge, (1996). 2.3

[10] K. Gopalsamy, Stability and oscillations in delay differential equations of population dynamics, Mathematics and its Applications, Kluwer Academic Publishers Group, Dordrecht, (1992). 2

[11] D.-Q. Jiang, C.-Y. Ji, X.-Y. Li, D. O'Regan, Analysis of autonomous Lotka-Volterra competition systems with random perturbation, J. Math. Anal. Appl., 390 (2012), 582-595. 2

[12] D.-Q. Jiang, N.-Z. Shi, X.-Y. Li, Global stability and stochastic permanence of a non-autonomous logistic equation with random perturbation, J. Math. Anal. Appl., 340 (2008), 588-597. 1, 2

[13] H. Kunita, Itô's stochastic calculus: its surprising power for applications, Stochastic Process. Appl., 120 (2010), 622 $652, .2 .7,2.1$

[14] D. Li, J.-A. Cui, G.-H. Song, Permanence and extinction for a single-species system with jump-diffusion, J. Math. Anal. Appl., 430 (2015), 438-464,. 1

[15] X.-Y. Li, A. Gray, D.-Q. Jiang, X.-R. Mao, Sufficient and necessary conditions of stochastic permanence and extinction for stochastic logistic populations under regime switching, J. Math. Anal. Appl., 376 (2011), 11-28. 1, 2

[16] X.-Y. Li, D.-Q. Jiang, X.-R. Mao, Population dynamical behavior of Lotka-Volterra system under regime switching, J. Comput. Appl. Math., 232 (2009), 427-448. 1

[17] X.-Y. Li, X.-R. Mao, Population dynamical behavior of non-autonomous Lotka-Volterra competitive system with random perturbation, Discrete Contin. Dyn. Syst., 24 (2009), 523-545. 1.1, 1, 1, 1, 2

[18] M. Y. Li, Z.-S. Shuai, Global-stability problem for coupled systems of differential equations on networks, J. Differential Equations, 248 (2010), 1-20. 2.2

[19] W.-X. Li, X.-Q. Zhang, C.-M. Zhang, A new method for exponential stability of coupled reaction-diffusion systems with mixed delays: combining Razumikhin method with graph theory, J. Franklin Inst., 352 (2015), 1169-1191. 4

[20] R. S. Lipster, A strong law of large numbers for local martingales, Stochastics, 3 (1980), 217-228. 2.7

[21] M. Liu, C.-Z. Bai, Analysis of a stochastic tri-trophic food-chain model with harvesting, J. Math. Biol., 73 (2016), 597-625. 1,4

[22] M. Liu, C.-Z. Bai, Dynamics of a stochastic one-prey two-predator model with Léry jumps, Appl. Math. Comput., 284 (2016), 308-321. 1

[23] M. Liu, C.-Z. Bai, Optimal harvesting of a stochastic delay competitive model, Discrete Contin. Dyn. Syst. Ser. B, 22 (2017), 1493-1508. 2, 2.3

[24] M. Liu, C.-Z. Bai, Optimal harvesting of a stochastic mutualism model with Lévy jumps, Appl. Math. Comput., 276 (2016), 301-309. 1

[25] M. Liu, C.-Z. Bai, Y. Jin, Population dynamical behavior of a two-predator one-prey stochastic model with time delay, Discrete Contin. Dyn. Syst., 37 (2017), 2513-2538. 1, 2.2, 2.3

[26] Q. Liu, Q.-M. Chen, Z.-H. Liu, Analysis on stochastic delay Lotka-Volterra systems driven by Lévy noise, Appl. Math. Comput., 235 (2014), 261-271. 2.6, 2.11, 4, 4

[27] M. Liu, M. Fan, Permanence of stochastic Lotka-Volterra systems, J. Nonlinear Sci., 27 (2017), 425-452. 1, 1

[28] M. Liu, M. Fan, Stability in distribution of a three-species stochastic cascade predator-prey system with time delays, IMA J. Appl. Math., 82 (2017), 396-423. 1, 2.3

[29] J.-L. Lv, K. Wang, Asymptotic properties of a stochastic predator-prey system with Holling II functional response, Commun. Nonlinear Sci. Numer. Simul., 16 (2011), 4037-4048. 1

[30] J.-L. Lv, K. Wang, X.-L. Zou, Remarks on stochastic permanence of population models, J. Math. Anal. Appl., 408 (2013), 561-571. 1

[31] P. S. Mandal, S. Abbas, M. Banerjee, A comparative study of deterministic and stochastic dynamics for a non-autonomous allelopathic phytoplankton model, Appl. Math. Comput., 238 (2014), 300-318. 1

[32] J. W. Moon, Counting labelled trees, From lectures delivered to the Twelfth Biennial Seminar of the Canadian Mathematical Congress, Vancouver, (1969), Canadian Mathematical Monographs, Canadian Mathematical Congress, Montreal, Que., (1970). 2.2

[33] S.-G. Peng, X.-H. Zhu, Necessary and sufficient condition for comparison theorem of 1-dimensional stochastic differential equations, Stochastic Process. Appl., 116 (2006), 370-380.2.1 
[34] H. Qiu, J.-L. Lv, K. Wang, Two types of permanence of a stochastic mutualism model, Adv. Difference Equ., 2013 (2013), 17 pages. 1

[35] S. J. Schreiber, M. Benaïm, K. A. S. Atchadé, Persistence in fluctuating environments, J. Math. Biol., 62 (2011), $655-683$. $1,1,1.2,1,2.10,4$

[36] H. L. Smith, H. R. Thieme, Dynamical systems and population persistence, Graduate Studies in Mathematics, American Mathematical Society, Providence, RI, (2011). 1

[37] R.-H. Tan, Z.-J. Liu, S.-L. Guo, H.-L. Xiang, On a nonautonomous competitive system subject to stochastic and impulsive perturbations, Appl. Math. Comput., 256 (2015), 702-714. 1, 2

[38] K. Tran, G. Yin, Stochastic competitive Lotka-Volterra ecosystems under partial observation: feedback controls for permanence and extinction, J. Franklin Inst., 351 (2014), 4039-4064. 1, 2

[39] M. Vasilova, M. Jovanović, Stochastic Gilpin-Ayala competition model with infinite delay. Appl. Math. Comput., 217 (2011), 4944-4959. 2

[40] G.-C. Wu, D. Baleanu, Discrete fractional logistic map and its chaos, Nonlinear Dynam., 75 (2014), 283-287. 4

[41] G.-C. Wu, D. Baleanu, H.-P. Xie, F.-L. Chen, Chaos synchronization of fractional chaotic maps based on the stability condition, Phys. A, 460 (2016), 374-383. 4

[42] F.-K. Wu, Y. Xu, Stochastic Lotka-Volterra population dynamics with infinite delay, SIAM J. Appl. Math., 70 (2009), 641-657. 2

[43] C.-M. Zhang, W.-X. Li, K. Wang, Graph-theoretic approach to stability of multi-group models with dispersal, Discrete Contin. Dyn. Syst. Ser. B, 20 (2015), 259-280. 4

[44] C.-M. Zhang, W.-X. Li, K. Wang, Graph-theoretic method on exponential synchronization of stochastic coupled networks with Markovian switching, Nonlinear Anal. Hybrid Syst., 15 (2015), 37-51. 4

[45] X.-H. Zhang, K. Wang, Asymptotic behavior of stochastic Gilpin-Ayala mutualism model with jumps, Electron. J. Differential Equations, 2013 (2013), 17 pages. 1, 4

[46] Y. Zhu, M. Liu, Permanence and extinction in a stochastic service-resource mutualism model, Appl. Math. Lett., 69 (2017), 1-7. 1 